Pacific

Journal of

Mathematics

\title{
CONVEX SOLUTIONS TO THE POWER-OF-MEAN CURVATURE FLOW
}

SHIBING CHEN

Volume $276 \quad$ No. 1

July 2015 


\title{
CONVEX SOLUTIONS TO THE POWER-OF-MEAN CURVATURE FLOW
}

\author{
SHIBING CHEN
}

We prove some estimates for convex ancient solutions (the existence time for the solution starts at $-\infty$ ) to the power-of-mean curvature flow, when the power is strictly greater than $\frac{1}{2}$. As an application, we prove that in dimension two, the blow-down of an entire convex translating solution, namely $u_{h}=\frac{1}{h} u\left(h^{\frac{1}{1+\alpha}} x\right)$, locally uniformly converges to $\frac{1}{1+\alpha}|x|^{1+\alpha}$ as $h \rightarrow \infty$. Another application is that for the generalized curve shortening flow (convex curve evolving in its normal direction with speed equal to a power of its curvature), if the convex compact ancient solution sweeps the whole space $\mathbb{R}^{2}$, it must be a shrinking circle. Otherwise the solution must be defined in a strip region.

\section{Introduction}

Classifying ancient convex solutions to mean curvature flow is very important in studying the singularities of mean curvature flow. Translating solutions arise as a special case of ancient solutions when one uses a proper procedure to blow up the mean convex flow near type II singular points, and general ancient solutions arise at general singularities. Some important progress was made by Wang [2011], and Daskalopoulos, Hamilton and Sesum [Daskalopoulos et al. 2010]. Wang proved that in dimension $n=2$, an entire convex translating solution to mean curvature flow must be rotationally symmetric in an appropriate coordinate system, which was a conjecture formulated explicitly by White [2000], but for $n \geq 3$ such solutions are not necessarily rotationally symmetric.

Wang also constructed some entire convex translating solutions with level sets neither spherical nor cylindrical in dimension greater or equal to 3. In the same paper, Wang also proved that if a convex ancient solution to the curve shortening flow sweeps the whole space $\mathbb{R}^{2}$, then it must be a shrinking circle — otherwise the convex ancient solution must be defined in a strip region, and he indeed constructed such solutions by a compactness argument. Daskalopoulos et al. [2010] showed that apart from the shrinking circle, the so called Angenent oval (a convex ancient

MSC2010: $35 \mathrm{~J} 60$.

Keywords: mean curvature flow, convexity, translating solution, ancient solution. 
solution of the curve shortening flow discovered by Angenent that decomposes into two translating solutions of the flow) is the only other embedded convex compact ancient solution of the curve shortening flow. That means that the corresponding curve shortening solution defined in a strip region constructed by Wang is exactly the "Angenent oval".

The power-of-mean curvature flow, in which a hypersurface evolves in its normal direction with speed equal to a power $\alpha$ of its mean curvature $H$, is well-studied [Andrews 1998; 2003; 2002; Schulze 2005; Chou and Zhu 2001; Sheng and Wu 2009]. Schulze [2005] called it $H^{\alpha}$-flow. In the following, we will also call the one dimensional power-of-curvature flow the generalized curve shortening flow. It would be very interesting if one could classify the ancient convex solutions. In this paper, we use the method developed in [Wang 2011] to study the geometric asymptotic behavior of ancient convex solutions to $H^{\alpha}$-flow. The general equation for $H^{\alpha}$-flow is

$$
\frac{\partial F}{\partial t}=-H^{\alpha} v
$$

where $F: M \times[0, T) \rightarrow \mathbb{R}^{n+1}$ is a time-dependent embedding of the evolving hypersurface, $v$ is the unit normal vector to the hypersurface $F(M, t)$ in $\mathbb{R}^{n+1}$, and $H$ is its mean curvature. If the evolving hypersurface can be represented as a graph of a function $u(x, t)$ over some domain in $\mathbb{R}^{n}$, then we can project the evolution equation to the $(n+1)$-st coordinate direction of $\mathbb{R}^{n+1}$ and the equation becomes

$$
u_{t}=\sqrt{1+|D u|^{2}}\left(\operatorname{div} \frac{D u}{\sqrt{1+|D u|^{2}}}\right)^{\alpha} .
$$

Then a translating solution to the $H^{\alpha}$-flow will satisfy the equation

$$
\sqrt{1+|D u|^{2}}\left(\operatorname{div} \frac{D u}{\sqrt{1+|D u|^{2}}}\right)^{\alpha}=1,
$$

which is equivalent to the special case $\sigma=1$ of the following:

$$
\begin{aligned}
L_{\sigma}(u) & =\left(\sqrt{\sigma+|D u|^{2}}\right)^{\frac{1}{\alpha}} \operatorname{div} \frac{D u}{\sqrt{\sigma+|D u|^{2}}} \\
& =\left(\sigma+|D u|^{2}\right)^{\frac{1}{2 \alpha}-\frac{1}{2}} \sum_{i, j=1}^{n}\left(\delta_{i j}-\frac{u_{i} u_{j}}{\sigma+|D u|^{2}}\right) u_{i j} \\
& =1,
\end{aligned}
$$

where $\sigma \in[0,1], \alpha \in\left(\frac{1}{2}, \infty\right]$ is a constant, $n=2$ is the dimension of $\mathbb{R}^{2}$. If $u$ is a convex solution of (3), then $u+t$, as a function of $(x, t) \in \mathbb{R}^{2} \times \mathbb{R}$, is a translating 
solution to the flow

$$
u_{t}=\sqrt{\sigma+|D u|^{2}}\left(\operatorname{div} \frac{D u}{\sqrt{\sigma+|D u|^{2}}}\right)^{\alpha} .
$$

When $\sigma=1$, Equation (4) is the nonparametric power-of-mean curvature flow. When $\sigma=0$, Equation (3) is the level set flow. That is, if $u$ is a solution of (3) with $\sigma=0$, then the level set $\{u=-t\}$, where $-\infty<t<-\inf u$, evolves by the power-of-mean curvature.

In the following we will assume $\sigma \in[0,1], \alpha \in\left(\frac{1}{2}, \infty\right]$, and the dimension $n=2$, although some of the estimates do hold in higher dimension. The main results of this paper are the following theorems.

Theorem 1. Let $u$ be an entire convex solution of (3). Let

$$
u_{h}(x)=h^{-1} u\left(h^{\frac{1}{1+\alpha}} x\right) .
$$

Then, $u_{h}$ locally uniformly converges to

$$
\frac{1}{1+\alpha}|x|^{1+\alpha} \quad \text { as } h \rightarrow \infty \text {. }
$$

Theorem 2. Let $u_{\sigma}$ be an entire convex solution of (3). Then,

$$
u_{0}(x)=\frac{1}{1+\alpha}|x|^{1+\alpha}
$$

up to a translation of the coordinate system. When $\sigma \in(0,1]$, if

$$
\left|D^{2} u(x)\right|=O\left(|x|^{\beta}\right) \quad \text { as }|x| \rightarrow \infty
$$

for some fixed constant $\beta$ satisfying $\beta<3 \alpha-2$, then $u_{\sigma}$ is rotationally symmetric after a proper translation of the coordinate system.

Corollary 3. A convex compact ancient solution to the generalized curve shortening flow which sweeps the whole space $\mathbb{R}^{2}$ must be a shrinking circle.

Remark 4. The condition $\alpha>\frac{1}{2}$ is necessary for our results. One can consider the translating solution $v(x)$ to (3) with $\sigma=1$ in one dimension. In fact, when $\alpha \leq \frac{1}{2}$, the translating solution $v(x)$ is a convex function defined on the entire real line [Chou and Zhu 2001, p. 28]. Then one can construct a function $u(x, y)=v(x)-y$, defined on the entire plane, and $u$ will satisfy (3) with $\sigma=0$; it is obviously not rotationally symmetric. We can also let $u(x, y)=v(x)$, which is an entire solution to (3) with $\sigma=1$, and it is not rotationally symmetric.

When the dimension is at least two, similar examples can be given: we can take an entire rotationally symmetric solution $v(x)$ to (3) with $n \geq 2$ and $\sigma=1$, and again let $u(x, y)=v(x)-y$ (here, $y$ is the $(n+1)$-st coordinate for $\left.\mathbb{R}^{n+1}\right)$. It is easy to see that $u$ will satisfy (3) with $n$ replaced by $n+1$ and $\sigma=0$, and the level set of $u$ is neither a sphere nor a cylinder. 
We would also like to point out that this elementary construction can be used to give a slight simplification of the proof of [Wang 2011, Theorem 2.1] (corresponding to our Corollary 10 for $\alpha=1$ ). Let $v_{\sigma}$ be an entire convex solution to (3) in dimension $n$ with $\sigma \in(0,1]$. Then $u(x, y)=v_{\sigma}(x)-\sqrt{\sigma} y$ will be an entire convex solution to (3) in dimension $n+1$ with $\sigma=0$. Hence if one has proved the estimate in Corollary 10 for $\sigma=0$ in all dimensions, the estimate for $\sigma \in(0,1]$ follows immediately from the above construction. The remainder of the paper is divided into four sections. Sections 2 and 3 contain the proof of Theorem 1 and the first part of Theorem 2. Section 4 is devoted to the proof of Corollary 10, and the last section completes the proof of Theorem 2 .

\section{Power growth estimate}

In this section, we prove a key estimate, which says that any entire convex solution $u$ to (3) must satisfy

$$
u(x) \leq C\left(1+|x|^{1+\alpha}\right),
$$

where the constant $C$ depends only on the upper bound of $u(0)$ and $|D u(0)|$. When $\alpha=1$, the estimate was proved by Wang [2011, Theorem 2.1]. To apply Wang's method, the main difficulty is that now the speed function is nonlinear in the curvature. We overcome this difficulty by further exploiting some elementary convexity properties.

For any constant $h>0$, we denote

$$
\begin{aligned}
\Gamma_{h} & =\left\{x \in \mathbb{R}^{n}: u(x)=h\right\}, \\
\Omega_{h} & =\left\{x \in \mathbb{R}^{n}: u(x)<h\right\},
\end{aligned}
$$

so that $\Gamma_{h}$ is the boundary of $\Omega_{h}$. Let $\kappa$ be the curvature of the level curve $\Gamma_{h}$. We have

$$
\begin{aligned}
L_{\sigma}(u) & =\left(\sigma+u_{\gamma}^{2}\right)^{\frac{1}{2 \alpha}-\frac{1}{2}}\left(\kappa u_{\gamma}+\frac{\sigma u_{\gamma \gamma}}{\sigma+u_{\gamma}^{2}}\right) \\
& \geq \kappa u_{\gamma}^{\frac{1}{\alpha}}=L_{0}(u),
\end{aligned}
$$

where $\gamma$ is the unit outward normal to $\Omega_{h}$, and $u_{\gamma \gamma}=\gamma_{i} \gamma_{j} u_{i j}$.

Before starting the proof of our main results, we recall a well known convergence result for the generalized curve shortening flow.

Lemma 5 [Andrews 2003, Theorems 1.3, 1.4, 1.5]. Let $\ell_{t}$ be a time-dependent family of closed curves in $\mathbb{R}^{2}$ evolving under the generalized curve shortening flow with $\alpha>\frac{1}{3}$. Suppose the initial curve $\ell_{0}$ is convex. Then the curve converges in finite time $T$ to a round point $P$ in the sense that $((1+\alpha)(T-t))^{-\frac{1}{1+\alpha}}\left(\ell_{t}-P\right)$ is asymptotic to the unit circle. 
Next, we prove a lemma which will be used to control the shape of the level set of a complete convex solution to (3).

Lemma 6. Let $u$ be a complete convex solution of (3). Suppose that $u(0)=0$ and that the infimum $\inf \left\{|x|: x \in \Gamma_{1}\right\}$ is attained at $x_{0}=(0,-\delta) \in \Gamma_{1}$ for some $\delta>0$ sufficiently small. Let $D_{1}$ be the projection of $\Gamma_{1}$ onto the axis $\left\{x_{2}=0\right\}$. Then, $D_{1}$ contains the interval $(-R, R)$, and when $\alpha \leq 1, R$ satisfies

$$
R \geq C_{1}\left(-\log \delta-C_{2}\right)^{\frac{\alpha}{\alpha+1}},
$$

where $C_{1}, C_{2}>0$ are independent of $\delta$; when $\alpha>1, R \geq C$ for some positive constant $C$.

The proof of this lemma follows that of [Wang 2011, Lemma 2.4] with minor modifications; the for reader's convenience, we give some details here.

Proof. First, we prove the lemma when $\frac{1}{2}<\alpha \leq 1$. Suppose that near $x_{0}, \Gamma_{1}$ is given by $x_{2}=g\left(x_{1}\right)$. Then, $g$ is a convex function satisfying $g(0)=-\delta$ and $g^{\prime}(0)=0$. Let $b>0$ be a constant such that $g^{\prime}(b)=1$. To prove (7), it suffices to prove

$$
b \geq C_{1}\left(-\log \delta-C_{2}\right)^{\frac{\alpha}{\alpha+1}} .
$$

For any $y=\left(y_{1}, y_{2}\right) \in \Gamma_{1}$, where $y_{1} \in[0, b]$, as in the proof of [Wang 2011, Lemma 2.4] we have

$$
u_{\gamma}(y) \geq \frac{\sqrt{1+g^{\prime 2}}}{y_{1} g^{\prime}-y_{2}},
$$

where $\gamma$ is the unit normal of the sublevel set $\Omega_{1}$. Since $L_{0} u \leq 1$, we have

$$
\frac{g^{\prime \prime}}{\left(1+g^{\prime 2}\right)^{\frac{3}{2}}} \frac{\left(1+g^{\prime 2}\right)^{\frac{1}{2 \alpha}}}{\left(y_{1} g^{\prime}-y^{2}\right)^{\frac{1}{\alpha}}} \leq \kappa u_{\gamma}^{\frac{1}{\alpha}} \leq 1,
$$

where $\kappa$ is the curvature of the level curve $\Gamma_{1}$. Hence,

$$
\begin{aligned}
g^{\prime \prime}\left(y_{1}\right) & \leq\left(1+g^{\prime 2}\right)^{\frac{3}{2}-\frac{1}{2 \alpha}}\left(y_{1} g^{\prime}-y_{2}\right)^{\frac{1}{\alpha}} \\
& \leq 10 y_{1}^{\frac{1}{\alpha}} g^{\prime}+10 \delta
\end{aligned}
$$

where $y_{2}=g\left(y_{1}\right)$ and $g^{\prime}\left(y_{1}\right) \leq 1$ for $y_{1} \in(0, b)$. The inequality from (11) to (12) is trivial when $y_{2} \geq 0$. When $y_{2} \leq 0$, since $\left|y_{2}\right| \leq \delta$, we have either $y_{1} g^{\prime} \leq \delta$ or $y_{1} g^{\prime}>\delta$. For the former we have

$$
\left(y_{1} g^{\prime}-y_{2}\right)^{\frac{1}{\alpha}} \leq(2 \delta)^{\frac{1}{\alpha}} \leq 4 \delta ;
$$

for the latter, since $g^{\prime}\left(y_{1}\right) \leq 1$, we have

$$
\left(y_{1} g^{\prime}-y_{2}\right)^{\frac{1}{\alpha}} \leq\left(2 y_{1} g^{\prime}\right)^{\frac{1}{\alpha}} \leq 4 y_{1}^{\frac{1}{\alpha}} g^{\prime} .
$$


We consider the equation

$$
\rho^{\prime \prime}(t)=10 t^{\frac{1}{\alpha}} \rho^{\prime}+10 \delta
$$

with initial conditions $\rho(0)=-\delta$ and $\rho^{\prime}(0)=0$. Then for $t \in(0, b)$, we have

$$
\rho^{\prime}(t)=10 \delta e^{\frac{10 \alpha}{\alpha+1} t^{\frac{\alpha+1}{\alpha}}} \int_{0}^{t} e^{-\frac{10 \alpha}{\alpha+1} s^{\frac{\alpha+1}{\alpha}}} d s .
$$

Since $\int_{0}^{\infty} e^{-\frac{10 \alpha}{\alpha+1} s^{\frac{\alpha+1}{\alpha}}} d s$ is bounded above by some constant $C$, we have

$$
\begin{aligned}
1 \leq \rho^{\prime}(b) & =10 \delta e^{\frac{10 \alpha}{\alpha+1} b^{\frac{\alpha+1}{\alpha}}} \int_{0}^{b} e^{-\frac{10 \alpha}{\alpha+1} s^{\frac{\alpha+1}{\alpha}}} d s \\
& \leq C_{1} \delta e^{\frac{10 \alpha}{\alpha+1} b^{\frac{\alpha+1}{\alpha}}},
\end{aligned}
$$

from which (8) follows.

When $\alpha>1$, the situation is different. First, we introduce a number $a$ such that $g^{\prime}(a)=\frac{1}{2}$. Then, we can follow the proof above until (11). For (12) the inequality becomes

$$
g^{\prime \prime}\left(y_{1}\right) \leq 10 y_{1}^{\frac{1}{\alpha}} g^{\prime}+10 \delta^{\frac{1}{\alpha}},
$$

for $y_{1} \in[a, b]$. Now (16) becomes

$$
e^{-\frac{10 \alpha}{\alpha+1} b^{\frac{\alpha+1}{\alpha}}} \rho^{\prime}(b)-e^{-\frac{10 \alpha}{\alpha+1} a^{\frac{\alpha+1}{\alpha}}} \rho^{\prime}(a) \leq C_{1} \delta^{\frac{1}{\alpha}} .
$$

Then, it is easy to see that when $\delta$ is small, $b \geq C$, for some fixed constant $C$.

Remark 7. When $\alpha \leq 1$, it follows from Lemma 6 that when $\delta$ is sufficiently small, by convexity and in view of Figure 1 , we have that $\Omega_{1}$ contains the shadowed region. Then it is easy to check that $\Omega_{1}$ contains an ellipse

$$
E=\left\{\begin{array}{l|l}
\left(x_{1}, x_{2}\right) \mid \frac{x_{1}^{2}}{\left(\frac{R}{6}\right)^{2}}+\frac{\left(x_{2}-\frac{7 \delta^{*}-5 \delta}{12}\right)^{2}}{\left(\frac{\delta^{*}+\delta}{4}\right)^{2}}=1
\end{array}\right\},
$$

where $\delta^{*}$ is a positive constant such that $u\left(0, \delta^{*}\right)=1$ and $R$ is defined in Lemma 5 .

When $\alpha>1$, if $\delta^{*}$ is very large, in the part $\left\{x: u(x) \leq 1, x_{1} \geq 0\right\}$, by convexity we can find an ellipse which has the length of short axis bounded from below and the length of long axis very large, and if we let the ellipse evolve under the generalized curve shortening flow, it will take time more than 1 for it to converge to a round point. When $\delta^{*}$ is less than some fixed constant, we need to consider two cases.

Case 1: The set $\{u \leq 1\}$ is not compact. In this case when we project $\{u(x)=1\}$ to the axis $\left\{x_{2}=0\right\}$, and denote the leftmost and rightmost points as $(-l, 0)$ and $(r, 0)$, respectively. Then either $l$ or $r$ is very large, which guarantees that one can 


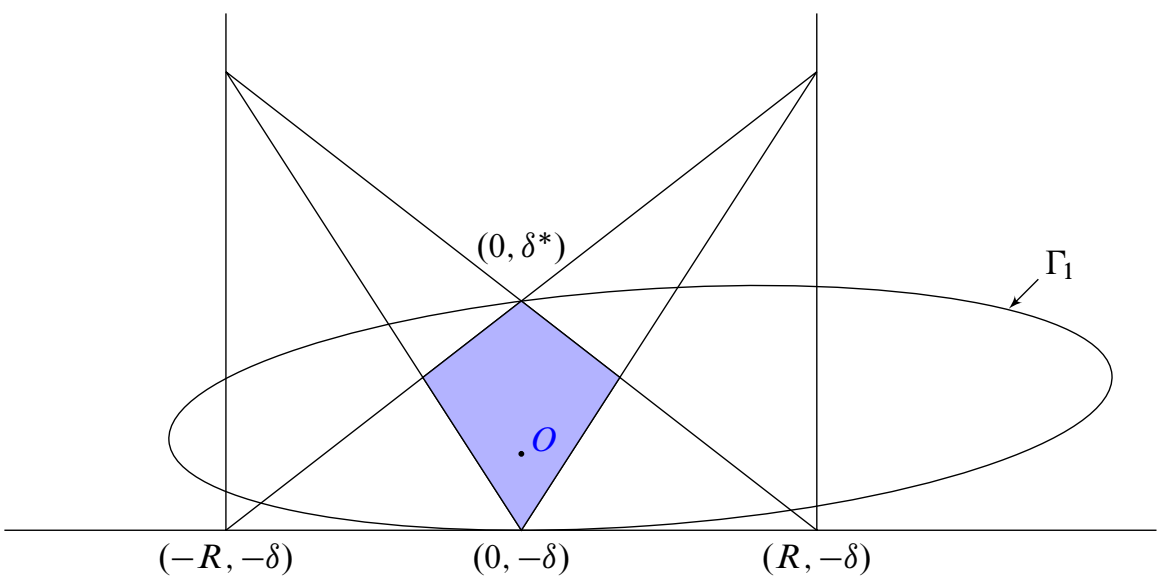

Figure 1. $\Gamma_{1}$ contains the shadow part.

still find an ellipse inside $\left\{x: u(x) \leq 1, x_{1} \leq 0\right\}$ (or $\left\{x: u(x) \leq 1, x_{1} \geq 0\right\}$ ) with the similar property as before.

Case 2: The set $\{u \leq 1\}$ is compact. For this case, we will always assume 0 is the minimum point of $u$, and $u(0)=0$. We claim that when $\delta$ is very small, for the purpose of the proof of Corollary 10, we can assume one of $l$ or $r$ is very large. Indeed, if the claim is not true, then we have a sequence of functions $u_{i}$ such that $\left\{u_{i} \leq 1\right\}$ has width bounded by some constant independent of $i$, and $\operatorname{dist}\left(0,\left\{u_{i} \leq 1\right\}\right) \rightarrow 0$ as $i \rightarrow \infty$. In view of the following proof of Corollary 10, we can assume $u_{i}$ satisfies (3) with $\sigma_{i} \rightarrow 0$. Then by passing to a subsequence, we can assume that $\left\{u_{i}=1\right\}$ converges to a convex curve $C_{0}$ in hausdorff distance. Let $C_{0}$ evolve under the generalized curve shortening flow; by Lemma 5, it will converge to a point $P$, but by the above discussion we see that $P$ is on $C_{0}$, which is clearly impossible. Once $l$ or $r$ is very large, we can find an ellipse with the similar property as in the case 1 .

Remark 8. One can also establish a similar lemma in higher dimensions, which says that $D_{1}$ (a convex set with dimension greater than 1) contains a ball centered at the origin with radius

$$
R \geq C_{n}(-\log \delta-C)^{\frac{\alpha}{\alpha+1}},
$$

where $C_{n}$ is a constant depending only on $n$ and $C$ is a positive constant independent of $\delta$. The proof can be reduced to the two dimensional case; for the details, refer to the proof of [Wang 2011, Lemma 2.6].

Lemma 9. Let $u$ be a complete convex solution of (3). Suppose $u(0)=0, \delta$ and $\delta^{*}$ are defined as in Lemma 6 and Remark 7. If $\delta$ and $\delta^{*}$ are sufficiently small, then $u$ is defined in a strip region. 
When $\alpha=1$, this lemma is proved by Wang [2011, Corollary 2.2]. The proof of Lemma 9 is based on a careful study of the shape of the level set of $u$. Before giving the proof, we will give an important corollary first.

Corollary 10. Let $u$ be an entire convex solution of (3) in $\mathbb{R}^{2}$, then

$$
u(x) \leq C\left(1+|x|^{1+\alpha}\right),
$$

where the constant $C$ depends only on the upper bound for $u(0)$ and $|D u(0)|$.

Proof. The proof of this Corollary follows the proof of [Wang 2011, Theorem 2.1]. We only record some necessary changes here. First, the rescaling $u_{h}(x)=\frac{1}{h} u\left(h^{\frac{1}{2}} x\right)$ used that proof should be replaced by $u_{h}(x)=\frac{1}{h} u\left(h^{\frac{1}{1+\alpha}} x\right)$. Note that $u_{h}$ solves (3) with $\sigma=\sigma_{h} \rightarrow 0$ as $h \rightarrow \infty$. Second, the ellipse used in that proof when applying the comparison argument should be replaced by the one discussed in Remark 7.

Proof of Lemma 9. By a rotation of coordinates we may assume that the axial directions of $E$ in Remark 7 coincide with those of the coordinate system. Let $M_{u}$ be the graph of $u$, which consists of two parts, $\mathcal{M}_{u}=\mathcal{M}^{+} \cup \mathcal{M}^{-}$, where

$$
\mathcal{M}^{+}=\left\{(x, u(x)) \in \mathbb{R}^{3}: \partial_{x_{2}} u \geq 0\right\} \quad \text { and } \quad \mathcal{M}^{-}=\left\{(x, u(x)) \in \mathbb{R}^{3}: \partial_{x_{2}} u \leq 0\right\} .
$$

Then $\mu^{ \pm}$can be represented as the graphs of functions $g^{ \pm}$of the form $x_{2}=$ $g^{ \pm}\left(x_{1}, x_{3}\right)$, for $\left(x_{1}, x_{3}\right) \in D$ where $D$ is the projection of $\mu_{u}$ onto the plane $\left\{x_{2}=0\right\}$. The functions $g^{+}$and $g^{-}$are concave and convex, respectively, and we have $x_{3}=u\left(x_{1}, g^{ \pm}\left(x_{1}, x_{3}\right)\right)$. Set

$$
g=g^{+}-g^{-} .
$$

Then $g$ is a positive, concave function on $D$, vanishing on $\partial D$. For any $h>0$ let $g_{h}\left(x_{1}\right)=g\left(x_{1}, h\right), g_{h}^{ \pm}\left(x_{1}\right)=g^{ \pm}\left(x_{1}, h\right)$, and $D_{h}=\left\{x_{1} \in \mathbb{R}^{1}:\left(x_{1}, h\right) \in D\right\}$. Then $g_{h}$ is a positive, concave function in $D_{h}$, vanishing on $\partial D_{h}$, and $D_{h}=\left(-\underline{a}_{h}, \bar{a}_{h}\right)$ is an interval containing the origin. Let $b_{h}=g_{h}(0)$. We consider the case $\sigma=0$ first.

Claim 1: Suppose $h$ is large, $g_{1}(0)=\delta^{*}+\delta$ is small, $b_{h} \leq 4$, and $\underline{a}_{h}, \bar{a}_{h} \geq b_{h}$. Then,

$$
\bar{a}_{h} \geq \frac{1}{1000} \frac{h}{b_{h}^{\alpha}} \text { for } \alpha \leq 1 \quad \text { and } \quad \bar{a}_{h} \geq \frac{1}{1000} \frac{h^{1 /(2 \alpha-1)}}{b_{h}^{1 /(2 \alpha-1)}} \text { for } \alpha>1 .
$$

Proof. Without loss of generality, we assume $\bar{a}_{h} \leq \underline{a}_{h}$. Let $U_{h}=\Omega_{h} \cap\left\{x_{1}>0\right\}$. By the convexity of $U_{h}$ and the assumption $\underline{a}_{h}, \bar{a}_{h} \geq b_{h}$, we have $\underline{a}_{s}, \bar{a}_{s} \geq \frac{1}{2} b_{h}$ for all $s \in\left(\frac{1}{2} h, h\right)$. Hence by the concavity of $g$,

$$
\left|\frac{d}{d x_{1}} g_{s}(0)\right| \leq 2 \quad \text { for } s \in\left(\frac{1}{2} h, h\right),
$$




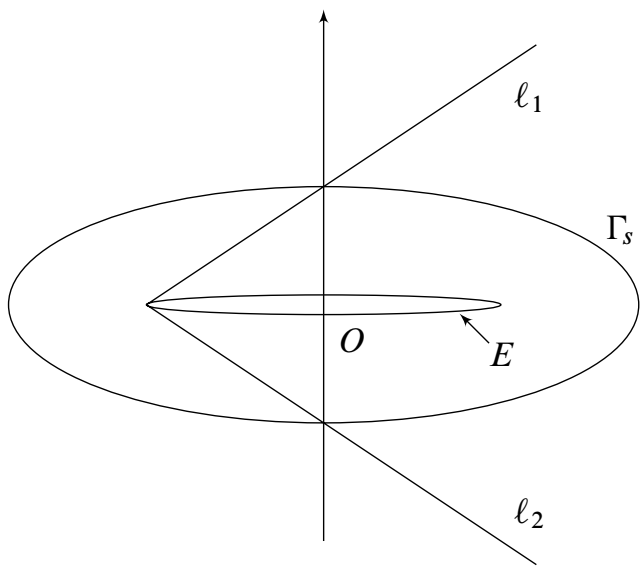

Figure 2. $\Gamma_{S} \cap\left\{x_{1}>0\right\}$ is trapped between two lines.

which means the arclength of the image of $\Gamma_{S} \cap\left\{x_{1}>0\right\}$ under the Gauss map is bigger then $\frac{\pi}{6}$. Notice that $\Omega_{1}$ contains $E$, which was defined in Remark 7 . When $\delta$ and $\delta^{*}$ are very small, $E$ is very thin and long. The center of $E$ is very close to the origin; in fact, for our purpose we can just pretend $E$ is centered at the origin. By convexity of $\Omega_{h}$ and in view of Figure 2, we see that $\Gamma_{s} \cap\left\{x_{1}>0\right\}$ is trapped between two lines $\ell_{1}$ and $\ell_{2}$, and the slopes of $\ell_{1}$ and $\ell_{2}$ are very close to 0 when $E$ is very long and thin. Then it is clear that the largest distance from the points on $\Gamma_{S} \cap\left\{x_{1}>0\right\}$ to the origin can not be bigger than $10 \bar{a}_{h}$. By convexity of $u$,

$$
u_{\gamma}(x) \geq \frac{h}{20 \bar{a}_{h}} \quad \text { for } x \in \Gamma_{S} \cap\left\{x_{1}>0\right\} .
$$

Since $\Gamma_{S} \cap\left\{x_{1}>0\right\}$ evolves under the generalized curve shortening flow, when $\alpha \leq 1$ we have the estimate

$$
\begin{aligned}
\frac{d}{d s}\left|U_{s}\right| & =\int_{\Gamma_{s} \cap\left\{x_{1}>0\right\}} \kappa^{\alpha} d \xi \\
& =\int_{\Gamma_{s} \cap\left\{x_{1}>0\right\}} u_{\gamma}^{\frac{1}{\alpha}-1} \kappa d \xi \\
& \geq \frac{1}{50}\left(\frac{h}{\bar{a}_{h}}\right)^{\frac{1}{\alpha}-1} \frac{\pi}{6},
\end{aligned}
$$

where from (20) to (21) we used the equation $\kappa u_{\gamma}^{\frac{1}{\alpha}}=1$. The claim follows by the simple fact that

$$
\frac{3}{2} b_{h} \bar{a}_{h} \geq\left|U_{h}\right| \geq \frac{1}{50}\left(\frac{h}{\bar{a}_{h}}\right)^{\frac{1}{\alpha}-1} \frac{\pi}{6} \frac{h}{2} .
$$

When $\alpha>1$, let $l_{s}$ denote the arclength of $\Gamma_{S} \cap\left\{x_{1}>0\right\}$. Then, by the above discussion, it is not hard to see that $l_{s} \approx C \bar{a}_{h}$. Then by a simple application of 
Jensen's inequality,

$$
\begin{aligned}
\frac{d}{d s}\left(\left|U_{s}\right|\right) & =\int_{\Gamma_{s} \cap\left\{x_{1}>0\right\}} \kappa^{\alpha} d \xi \\
& =l_{s} \int_{\Gamma_{s} \cap\left\{x_{1}>0\right\}} \kappa^{\alpha} \frac{1}{l_{s}} d \xi \\
& \geq l_{s}\left(\int_{\Gamma_{s} \cap\left\{x_{1}>0\right\}} \frac{\kappa}{l_{s}} d \xi\right)^{\alpha} \geq C l_{s}^{1-\alpha} \geq C \bar{a}_{h}^{1-\alpha} .
\end{aligned}
$$

Again by the simple fact that $\frac{3}{2} b_{h} \bar{a}_{h} \geq\left|U_{h}\right|$, we can complete the proof in the same way as in the previous case.

From here until (55) we will prove the lemma for the case $\frac{1}{2}<\alpha \leq 1$, and then we will give the details for the case $\alpha>1$.

Claim 2: Let $h_{k}=2^{k}, \bar{a}_{k}=\bar{a}_{h_{k}}, b_{k}=b_{h_{k}}, g_{k}=g_{h_{k}}$, and $D_{k}=D_{h_{k}}$. Then,

$$
g_{k}(0) \leq g_{k-1}(0)+C_{0} 2^{-k / C} \text { for all } k \text { large, }
$$

where $C_{0}$ is a fixed constant and $C$ depends only on $\alpha$.

Lemma 9 follows from Claims 1 and 2 in the following way. Let the convex set $P$ be the projection of the graph of $g$ onto the plane $\left\{x_{3}=0\right\}$. By Claim 2 and the fact that $P$ contains $x_{1}$-axis (it follows from Claim 1), $P$ must equal $I \times \mathbb{R}$ for some interval

$$
I \subset\left[0, \lim _{k \rightarrow \infty} g_{k}(0)\right]
$$

Then, by (19), $\mu_{u}$ is also contained in a strip region as stated in Lemma 9 .

Proof of Claim 2. To prove (23), observe that since $g$ is positive and concave,

$$
g_{k}(0) \leq h_{k} g_{0}(0) \leq 2^{k}\left(\delta+\delta^{*}\right)
$$

Hence, we can start from sufficiently large $k_{0}$, satisfying $g_{k_{0}}(0) \leq 1$ and

$$
g_{k_{0}}+C_{0} \sum_{j=k_{0}}^{\infty} 2^{-j / C} \leq 2 .
$$

Suppose (23) holds up to $k$. Then by (24), we have $g_{k}(0) \leq 2$. By the concavity of $g$ and and the fact that $g \geq 0$, we have $g_{k+1}(0) \leq 2 g_{k}(0) \leq 4$. By Claim 1, we have $\bar{a}_{k+1} \geq \frac{1}{10000} h_{k}$. To prove (23) at $k+1$,

$$
\begin{aligned}
& L_{k}=\left\{x_{1} \in \mathbb{R}^{1}:-\frac{C_{1}}{4} h_{k}<x_{1}<\frac{C_{1}}{4} h_{k}\right\}, \quad C_{1}=\frac{1}{10000}, \\
& Q_{k}=L_{k} \times\left[h_{k}, h_{k+1}\right] \subset D .
\end{aligned}
$$


Since $g>0$ and $g$ is concave, we have the estimates

$$
\begin{aligned}
g\left(x_{1}, h\right) & \leq 8, \\
\left|\partial_{h} g\left(x_{1}, h\right)\right| & \leq \frac{16}{h_{k}}, \\
\left|\partial_{x_{1}} g\left(x_{1}, h\right)\right| & \leq \frac{16}{h_{k}} \quad \text { for all }\left(x_{1}, h\right) \in Q_{k} .
\end{aligned}
$$

Let $\mathscr{X}^{ \pm}=\left\{\left(x_{1}, h\right) \in Q_{k}:\left|\partial_{x_{1} x_{1}} g^{ \pm}\left(x_{1}, h\right)\right| \geq h_{k}^{-\beta}\right\}$, where $\beta$ is chosen such that $\frac{1}{\alpha}<\beta<2$. For any $h \in\left(h_{k}, h_{k+1}\right)$, by (29),

$$
\begin{aligned}
\left|\left\{x_{1} \in L_{k}:\left(x_{1}, h\right) \in \mathscr{X}^{+}\right\}\right| h_{k}^{-\beta} & \leq \int_{L_{k}} \partial_{x_{1} x_{1}} g^{+} \\
& \leq \int_{L_{k}} \partial_{x_{1} x_{1}} g \\
& \leq 2 \sup _{L_{k}}\left|\partial_{x_{1}} g\right| \\
& \leq \frac{C}{h_{k}} .
\end{aligned}
$$

So, $\left|\mathscr{Q}^{+}\right| \leq C h_{k}^{\beta}$. Similarly, we have $\left|\mathscr{L}^{-}\right| \leq C h_{k}^{\beta}$.

For any given $y_{1} \in L_{k}$, let $\mathscr{X}_{y_{1}}^{ \pm}=\mathscr{X}^{+} \cap\left\{x_{1}=y_{1}\right\}$. Then, by the estimate above, there is a set $\widetilde{L}^{ \pm} \subset L_{k}$ with measure

$$
\left|\widetilde{L}^{ \pm}\right| \leq C h_{k}^{\beta / 2},
$$

such that for any $y_{1} \in L_{k}-\widetilde{L}^{ \pm}$, we have $\left|\mathscr{X}_{y_{1}}^{ \pm}\right| \leq h_{k}^{\beta} / 2$. When $k$ is large, we can always find $y_{1}=C h^{\beta / 2} \in L_{k}-\widetilde{L}^{ \pm}$, where the constant $C$ is under control. For such $y_{1}$, we have

$$
\begin{aligned}
g\left(y_{1}, h_{k+1}\right) & -g\left(y_{1}, h_{k}\right) \\
= & g^{+}\left(y_{1}, h_{k+1}\right)-g^{+}\left(y_{1}, h_{k}\right)+\left|g^{-}\left(y_{1}, h_{k+1}\right)-g^{-}\left(y_{1}, h_{k}\right)\right| .
\end{aligned}
$$

In the following, we will estimate $g^{+}\left(y_{1}, h_{k+1}\right)-g^{+}\left(y_{1}, h_{k}\right)$. The estimate for $\left|g^{-}\left(y_{1}, h_{k+1}\right)-g^{-}\left(y_{1}, h_{k}\right)\right|$ is analogous. By the same reason as that for [Wang 2011, §2.21], we have

$$
\left\{\begin{aligned}
\left(\partial_{h} g^{+}\right)^{-1} & =\left(1+\varepsilon_{1}\right) u_{\gamma} \\
\partial_{x_{1} x_{1}} g^{+} & =\left(1+\varepsilon_{2}\right) \kappa
\end{aligned}\right.
$$

Then, by the equation $u_{\gamma}^{\frac{1}{\alpha}} \kappa=1$, we have

$$
\partial_{h} g_{h}^{+}\left(y_{1}, h\right) \leq C\left(\partial_{x_{1} x_{1}} g^{+}\right)^{\alpha} \leq C h_{k}^{-\beta \alpha} .
$$


Now,

$$
\begin{aligned}
g^{+}\left(y_{1}, h_{k+1}\right)-g^{+}\left(y_{1}, h_{k}\right) & =\int_{h_{k}}^{h_{k+1}} \partial_{h} g^{+}\left(y_{1}, h\right) d h \\
& =\int_{\mathscr{X}_{y_{1}}^{+}} \partial_{h} g^{+}\left(y_{1}, h\right) d h+\int_{\left[h_{k}, h_{k+1}\right]-\mathscr{X}_{y_{1}}^{+}} d h \\
& \leq C_{1} h_{k}^{\frac{\beta}{2}} \frac{1}{h_{k}}+C_{2} h_{k}^{-\beta \alpha} h_{k} .
\end{aligned}
$$

Recall that $\beta$ satisfies $\frac{1}{\alpha}<\beta<2$, and we have $\eta:=\min \left\{1-\frac{\beta}{2}, \beta \alpha-1\right\}>0$. From (34) and (39), we have the estimate

$$
g\left(y_{1}, h_{k+1}\right)-g\left(y_{1}, h_{k}\right) \leq \frac{C}{h_{k}^{\eta}},
$$

for some fixed constant $C$. Then, we will assume $\partial_{x_{1}} g\left(0, h_{k}\right)<0$ (otherwise we can replace $x_{1}$ by $-x_{1}$ ); therefore, by the above estimate,

$$
g\left(y_{1}, h_{k+1}\right) \leq g\left(y_{1}, h_{k}\right)+\frac{C}{h_{k}^{\eta}} \leq g\left(0, h_{k}\right)+\frac{C}{h_{k}^{\eta}} .
$$

Since $g$ is positive, concave, and defined on $\left[0, \bar{a}_{k+1}\right]$, with $\bar{a}_{k+1} \geq C h_{k+1}$,

$$
\frac{g_{k+1}(0)}{g_{k+1}\left(y_{1}\right)} \leq \frac{\bar{a}_{k+1}}{\bar{a}_{k+1}-y_{1}} \leq 1+C h_{k+1}^{\frac{\beta}{2}-1} \text {. }
$$

Therefore, by the two estimates above,

$$
g_{k+1}(0) \leq g_{k}(0)+C h_{k}^{-\eta},
$$

which implies (23) immediately.

For the proof of Lemma 6 when $\sigma \in(0,1]$, we need to use (5) and (6). In fact, by (6) we see that $\Gamma_{h}$ is moving at a velocity greater than or equal to its curvature to the power $\alpha$. Hence, we still have the lower bound of $\frac{d}{d s}\left(\left|U_{s}\right|\right)$ as in the proof of Claim 1. Then we can follow the above proof for the case $\sigma=0$ until (37), replacing the equalities " $=$ " in (20) and (21) with inequalities " $\geq$ ". As in [Wang 2011], when $\sigma=0$, in order to control the second integral in (38) we used the equation $\kappa u_{\gamma}^{1 / \alpha}=1$. But when $\sigma \neq 0$, by (28) and (35) we have

$$
u_{\gamma} \geq C\left(\partial_{h} g^{+}\right)^{-1} \geq C h_{k} \text {. }
$$

Hence, we may assume that $u_{\gamma}$ is as large as we want, which means that in formula (5), the only important extra term is

$$
\left(\sigma+u_{\gamma}^{2}\right)^{\frac{1}{2 \alpha}-\frac{1}{2}} \frac{\sigma u_{\gamma \gamma}}{\sigma+u_{\gamma}^{2}}
$$


To handle this term, we divide the integral (39) into three parts:

$$
\begin{aligned}
g^{+}\left(y_{1}, h_{k+1}\right)-g+\left(y_{1}, h_{k}\right) & =\int_{h_{k}}^{h_{k+1}} \partial_{h} g^{+}\left(y_{1}, h\right) d h \\
& =\left(\int_{I_{1}}+\int_{I_{2}}+\int_{I_{3}}\right) \partial_{h} g^{+}\left(y_{1}, h\right) d h,
\end{aligned}
$$

where

$$
\begin{aligned}
& I_{1}=\mathscr{X}_{y_{1}}^{+}, \\
& I_{2}=\left\{h \in\left[h_{k}, h_{k+1}\right]-I_{1}:\left(\sigma+u_{\gamma}^{2}\right)^{\frac{1}{2 \alpha}-\frac{1}{2}} \frac{\sigma u_{\gamma \gamma}}{\sigma+u_{\gamma}^{2}} \leq \frac{1}{2}\right\}, \\
& I_{3}=\left[h_{k}, h_{k+1}\right]-I_{1} \cup I_{2} .
\end{aligned}
$$

For the first integral, we can do exactly the same thing as we have done from (38) to (39), namely,

$$
\int_{I_{1}} \partial_{h} g^{+}\left(y_{1}, h\right) d h \leq \frac{C}{h_{k}} h_{k}^{\frac{\beta}{2}}=C h_{k}^{\frac{\beta}{2}-1} .
$$

Note that the power $\frac{\beta}{2}-1$ is a negative number.

Then we estimate the second integral, note that when $\left(y_{1}, h\right) \in I_{2}$, we have

$$
\left(\sigma+u_{\gamma}^{2}\right)^{\frac{1}{2 \alpha}} \kappa u_{\gamma} \geq \frac{1}{2} \text {. }
$$

By (40) $u_{\gamma}$ is large, so we have $\kappa u_{\gamma}^{\frac{1}{\alpha}} \geq \frac{1}{4}$, hence by (35) we have

$$
\partial_{h} g^{+} \leq C\left(\partial_{x_{1} x_{1}} g^{+}\right)^{\alpha} \leq C h_{k}^{-\alpha \beta} .
$$

Therefore,

$$
\int_{I_{2}} \partial_{h} g^{+}\left(y_{1}, h\right) d h \leq C h_{k}^{-\beta \alpha} h_{k}=C h_{k}^{1-\beta \alpha} .
$$

Note that $1-\beta \alpha$ is a negative number. Observe that we can assume $I_{2}$ is on the right hand side of $I_{3}$, since by the concavity of $g^{+}$we know that when $h \geq \inf I_{2}$, $\partial_{h} g^{+}\left(y_{1}, h\right)$ will satisfy the estimate (46).

For the third integral, notice that by the same argument as that for [Wang 2011, $\S 2.24]$,

$$
\left\{\begin{array}{l}
u_{\gamma}\left(y_{1}, h\right)=u_{x_{2}}\left(1+\varepsilon_{1}\right), \\
u_{\gamma \gamma}\left(y_{1}, h\right)=u_{x_{2} x_{2}}\left(1+\varepsilon_{2}\right)+\varepsilon_{3} u_{x_{2}} .
\end{array}\right.
$$

Hence, by (47),

$$
\left(\sigma+u_{x_{2}}^{2}\right)^{\frac{1}{2 \alpha}-\frac{1}{2}} \frac{\sigma u_{x_{2} x_{2}}}{\sigma+u_{\gamma}^{2}} \geq \frac{1}{3} .
$$


Since $\sigma \in[0,1]$ and $u_{\gamma}$ is large, we have

$$
u^{\prime \prime}=u_{x_{2} x_{2}} \geq \frac{1}{4}\left(u^{\prime}\right)^{3-\frac{1}{\alpha}} .
$$

By differentiating the equation $u\left(x_{1}, g^{+}\left(x_{1}, h\right)\right)=h$ twice with respect to $h$,

$$
\left(g^{+}\right)^{\prime \prime}=-u^{\prime \prime}\left(g^{+}\right)^{\prime 3} \leq-\frac{1}{4}\left(g^{+}\right)^{\prime \frac{1}{\alpha}-3}\left(g^{+}\right)^{\prime 3}=-\frac{1}{4}\left(g^{+}\right)^{\prime \frac{1}{\alpha}} .
$$

Note that (50) is for points with corresponding $h \in I_{3}$. By the discussion after (46) we only need to estimate

$$
\int_{\left[h_{k}+h_{k}^{\frac{\beta+2}{4}}, \inf I_{2}\right]}\left(g^{+}\right)^{\prime} d h .
$$

Therefore, by (50) and noticing that $\left(g^{+}\right)^{\prime} \geq 0$,

$$
\frac{\alpha}{\alpha-1}\left(g^{+}\right)^{\prime \frac{\alpha-1}{\alpha}}(h) \leq \frac{\alpha}{\alpha-1}\left(g^{+}\right)^{\prime \frac{\alpha-1}{\alpha}}\left(h_{k}\right)-\frac{1}{4}\left|I_{3} \cap\left[h_{k}, h\right]\right| .
$$

Hence, when $h \in\left[h_{k}^{(\beta+2) / 4}, \inf I_{2}\right]$,

$$
\left(g^{+}\right)^{\prime}(h) \leq\left(\left(g^{+}\right)^{\prime \frac{\alpha-1}{\alpha}}\left(h_{k}\right)+C\left(h-h_{k}\right)\right)^{\frac{\alpha}{\alpha-1}} .
$$

Finally,

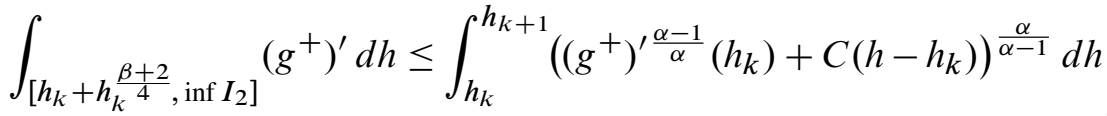

$$
\begin{aligned}
& \leq\left.\frac{\alpha-1}{2 \alpha-1}\left(\left(g^{+}\right)^{\prime \frac{\alpha-1}{\alpha}}\left(h_{k}\right)+C\left(h-h_{k}\right)\right)^{\frac{\alpha}{\alpha-1}+1}\right|_{h_{k}} ^{2 h_{k}} \\
& \leq C\left(g^{+}\right)^{\prime \frac{2 \alpha-1}{\alpha}} \leq C h_{k}^{\frac{1-2 \alpha}{\alpha}} \text {. }
\end{aligned}
$$

Note that $\frac{1-2 \alpha}{\alpha}<0$ when $\alpha>\frac{1}{2}$, so we can complete the proof as in the $\sigma=0$ case.

When $\alpha>1$, we need to choose the constants and exponents more carefully. First of all, in view of the Lemma 9 for $\alpha>1$, in order to have properties (35) and (47), we need only to replace the number 2 in (24) with some number much smaller than the constant $C$ in Lemma 9. The definition of $L_{k}$ in (25) should be modified to

$$
L_{k}=\left\{x_{1} \in \mathbb{R}^{1}:-\frac{C_{1}}{4} h_{k}^{\frac{1}{2 \alpha-1}}<x_{1}<\frac{C_{1}}{4} h_{k}^{\frac{1}{2 \alpha-1}}\right\}, \quad C_{1}=\frac{1}{10000},
$$

and the definition of $Q_{k}$ in (26) remains the same. It is easy to see that we still have the estimates (27)-(28), but (29) becomes

$$
\left|\partial_{x_{1}} g\left(x_{1}, h\right)\right| \leq 16 h_{k}^{-\frac{1}{2 \alpha-1}} \text { for all }\left(x_{1}, h\right) \in Q_{k} .
$$

Then for the definition of

$$
\mathscr{X}^{ \pm}=\left\{\left(x_{1}, h\right) \in Q_{k}:\left|\partial_{x_{1} x_{1}} g^{ \pm}\left(x_{1}, h\right)\right| \geq h_{k}^{-\beta}\right\},
$$


we need to choose the exponent $\beta$ so that $\frac{1}{\alpha}<\beta<\frac{2}{2 \alpha-1}$. By doing the same computation as (30)-(32),

$$
\left|\left\{x_{1} \in L_{k}:\left(x_{1}, h\right) \in \mathscr{L}^{+}\right\}\right| h_{k}^{-\beta} \leq \int_{L_{k}} \partial_{x_{1} x_{1}} g^{+} \leq C h_{k}^{-\frac{1}{2 \alpha-1}} .
$$

Hence,

$$
\left|\mathscr{Q}^{+}\right| \leq C h_{k}^{1+\beta-\frac{1}{2 \alpha-1}} \quad \text { and } \quad\left|\mathscr{Q}^{-}\right| \leq C h_{k}^{1+\beta-\frac{1}{2 \alpha-1}} .
$$

Then, by the above estimate there is a set $\widetilde{L}^{ \pm} \subset L_{k}$ with measure

$$
\left|\widetilde{L}^{ \pm}\right| \leq C h_{k}^{\beta+\varepsilon-\frac{1}{2 \alpha-1}}
$$

such that for any $y_{1} \in L_{k}-\widetilde{L}^{ \pm}$, we have $\left|\mathscr{X}_{y_{1}}^{ \pm}\right| \leq h_{k}^{1-\varepsilon}$, where $\varepsilon$ is chosen such that $\beta+\varepsilon<\frac{2}{2 \alpha-1}$. Now, (35)-(38) remain the same, and (39) becomes

$$
g^{+}\left(y_{1}, h_{k+1}\right)-g^{+}\left(y_{1}, h_{k}\right) \leq C_{1} h_{k}^{1-\varepsilon} \frac{1}{h_{k}}+C_{2} h_{k}^{-\beta \alpha} h_{k} .
$$

By the choice of $\beta$, all the exponents of $h_{k}$ are negative. We do not need to change anything from (40) to (49). Finally from (50) we need to replace the computation in the case $\alpha \leq 1$ with the following computation.

First, we have $\left(g^{+}\right)^{\prime \prime} \leq-\frac{1}{4}\left(g^{+}\right)^{\prime} \frac{1}{\alpha} \leq-\frac{1}{4}\left(g^{+}\right)^{\prime}$, and we only need to bound

$$
\int_{\left[h_{k}+h_{k}^{1-\varepsilon / 2}, \inf I_{2}\right]}\left(g^{+}\right)^{\prime} d h .
$$

Note that $\left(g^{+}\right)^{\prime} \geq 0$. By integrating the above differential inequality, we have

$$
\left(g^{+}\right)^{\prime}(h) \leq\left(g^{+}\right)^{\prime}\left(h_{k}\right) e^{-\frac{1}{4}\left|I_{3}\right|} \leq\left(g^{+}\right)^{\prime}\left(h_{k}\right) e^{\frac{1}{8}\left(h-h_{k}\right)}
$$

when $h \in\left[h_{k}+h_{k}^{1-\varepsilon / 2}\right.$, inf $\left.I_{2}\right]$. Therefore, we have

$$
\begin{aligned}
\int_{\left[h_{k}+h_{k}^{1-\varepsilon / 2}, \inf I_{2}\right]}\left(g^{+}\right)^{\prime} d h & \leq \int_{h_{k}}^{h_{k+1}}\left(g^{+}\right)^{\prime}\left(h_{k}\right) e^{\frac{1}{8}\left(h-h_{k}\right)} d h \\
& \leq C\left(g^{+}\right)^{\prime}\left(h_{k}\right) \leq \frac{C}{h_{k}}
\end{aligned}
$$

\section{Blow-down of an entire convex ancient solutions} converges to a power function

In this section we prove that the blow-down of an entire convex solution to (3) converges to a power function.

Proof of Theorem 1 and the first part of Theorem 2. First, we prove that there is a subsequence of $u_{h}$ converging to $\frac{1}{1+\alpha}|x|^{1+\alpha}$, where $u_{h}(x)=h^{-1} u\left(h^{\frac{1}{1+\alpha}} x\right)$. 
By adding a constant we may suppose $u(0)=0$. Let $x_{n+1}=a \cdot x$ be the equation of the tangent plane of $u$ at 0 . By Corollary 10 and the convexity of $u$ we have

$$
a \cdot x \leq u(x) \leq C\left(1+|x|^{1+\alpha}\right) .
$$

Hence,

$$
h^{-\frac{\alpha}{1+\alpha}} a \cdot x \leq u_{h}(x) \leq C\left(\frac{1}{h}+|x|^{1+\alpha}\right) .
$$

By convexity, $D u_{h}$ is locally uniformly bounded. Hence, $u_{h}$ subconverges to a convex function $u_{0}$ which satisfies $u_{0}(0)=0$, and

$$
0 \leq u_{0}(x) \leq C|x|^{1+\alpha} .
$$

It is easy to check that $u_{0}$ is an entire convex viscosity solution to (3) with $\sigma=0$, and the comparison principle holds on any bounded domain.

Now we will prove that $\left\{u_{0}(x)=0\right\}=\{0\}$. In fact, if $\left\{x: u_{0}(x)=0\right\}$ is a bounded set, then $\left\{u_{0}(x)=h\right\}$ is a closed, bounded convex curve which evolves under the generalized curve shortening flow; from [Andrews 1998] it follows that $\left\{u_{0}(x)=0\right\}=\{0\}$. If $\left\{u_{0}(x)=0\right\}$ contains a straight line, say the line $\{(t, 0): t \in \mathbb{R}\}$, then by convexity, $u$ is independent of $x_{1}$, which is impossible. So we only need to rule out the possibility that $\left\{u_{0}(x)=0\right\}$ contains a ray but no straight lines. In this case, for fixed $h>0$, we can find an ellipse $E$ inside $\left\{u_{0}(x)<h\right\}$, with the short axis bounded from below by a constant depending only on $h$ and with the long axis as long as we want (one only needs to look at the asymptotic cone of $\left\{u_{0}(x)=h\right\}$ ), but since $\left\{u_{0}(x)=h\right\}$ evolves under the generalized curve shortening flow and $E \subset\left\{u_{0}(x) \leq h\right\}$, this is impossible by comparison principle.

Then since $\left\{u_{0}(x)=0\right\}=\{0\}, \Gamma_{1, u_{0}}=\left\{u_{0}(x)=1\right\}$ is a bounded convex curve, and the level set $\left\{u_{0}(x)=-t\right\}$ evolves under the generalized curve shortening flow, with time $t \in(-\infty, 0)$. From [Andrews 1998; 2003] we have the following asymptotic behavior of the convex solution $u_{0}$ of $L_{0} u=1$ :

$$
u_{0}(x)=\frac{1}{1+\alpha}|x|^{1+\alpha}+\varphi(x),
$$

where $\varphi(x)=o\left(|x|^{\alpha+1}\right)$ for $x \neq 0$ near the origin. In fact, if the initial level curve is in a sufficiently small neighborhood of circle, by Lemma $13,|\varphi(x)| \leq C|x|^{1+\alpha+\eta}$ for some small positive $\eta$, where $C$ is a constant depending only on the initial closeness to the circle. Hence, given any $\epsilon>0$, for sufficiently small $h^{\prime}>0$,

$$
B_{(1-\epsilon) r}(0) \subset \Omega_{h^{\prime}, u_{0}} \subset B_{(1+\epsilon) r}(0),
$$

where $r=\left((1+\alpha) h^{\prime}\right)^{\frac{1}{1+\alpha}}$. Hence, there is a sequence $h_{m} \rightarrow \infty$ such that

where

$$
B_{\left(1-\frac{1}{m}\right) r_{m, i}}(0) \subset \Omega_{h_{m}, u} \subset B_{\left(1+\frac{1}{m}\right) r_{m, i}}(0),
$$

$$
r_{m, i}=\left((1+\alpha) i h_{m}\right)^{\frac{1}{1+\alpha}}, \quad i=1, \ldots, m .
$$


Then $u_{h_{m}}$ subconverges to $\frac{1}{1+\alpha}|x|^{1+\alpha}$.

Since $u_{0}$ is an entire convex solution to $L_{0} u=1$ (we still use the notation $u_{0}$, but it means an arbitrary entire convex solution), from the above argument, we can find a sequence $h_{m}$ such that

$$
u_{0 h_{m}}(x)=\frac{1}{h_{m}} u_{0}\left(h_{m}^{\frac{1}{1+\alpha}} x\right)
$$

locally uniformly converges to $\frac{1}{1+\alpha}|x|^{1+\alpha}$. Hence, the sublevel set $\Omega \frac{1}{1+\alpha}, u_{0 h_{m}}$ satisfies

$$
B_{1-\epsilon_{m}}(0) \subset \Omega \frac{1}{1+\alpha}, u_{0 h_{m}} \subset B_{1+\epsilon_{m}}(0),
$$

where $\epsilon_{m} \rightarrow 0$ as $m \rightarrow \infty$. By the discussion below (56),

$$
u_{0 h_{m}}(x)=\frac{1}{1+\alpha}|x|^{1+\alpha}+\varphi(x),
$$

where $|\varphi(x)| \leq C|x|^{1+\alpha+\eta}$ for some fixed small positive $\eta$, and the constant $C$ is independent of $m$. Replacing $x$ by $h_{m}^{-1 /(1+\alpha)} x$ in the asymptotic formula above,

$$
u_{0}(x)=\frac{1}{1+\alpha}|x|^{1+\alpha}+h_{m} \varphi\left(h_{m}^{-\frac{1}{1+\alpha}} x\right) \text {, }
$$

where for any fixed $x, h_{m} \varphi\left(h_{m}^{-\frac{1}{1+\alpha}} x\right) \rightarrow 0$. Hence $u_{0}(x)=\frac{1}{1+\alpha}|x|^{1+\alpha}$. So we have proved Theorem 1 and the first part of Theorem 2 .

\section{One-dimensional entire convex ancient solution must be a shrinking circle}

This section is devoted to the proof of Corollary 3, which is completed by combining the following lemma (corresponding to [Wang 2011, Lemma 4.1]) and Theorem 2.

Lemma 11. Let $\Omega$ be a smooth, bounded, convex domain in $\mathbb{R}^{2}$. Let $u$ be the solution of (3) with $\sigma=0$, vanishing on $\partial \Omega$. Then for any constant $h$ satisfying $\inf _{\Omega} u<h<0$, the level set $\Gamma_{h, u}=\{u=h\}$ is convex. Moreover, $\log (-u)$ is a concave function.

Proof. Observe that $\varphi:=-\log (-u)$ satisfies

$$
|D \varphi|^{\frac{1}{\alpha}-1} \sum_{i, j=1}^{2}\left(\delta_{i j}-\frac{\varphi_{i} \varphi_{j}}{|D \varphi|^{2}}\right) \varphi_{i j}=e^{\frac{1}{\alpha} \varphi} .
$$

Since $\varphi(x) \rightarrow+\infty$ as $x \rightarrow \partial \Omega$, [Kawohl 1985, Theorem 3.13] implies that $\varphi$ is convex.

With the previous lemma and [Wang 2011, Lemma 4.4], we know that any convex compact ancient solution to the generalized curve shortening flow can be represented as a convex solution $u$ to (3) with $\sigma=0$, and if the solution to the flow 
sweeps the whole space, then the corresponding $u$ will be an entire solution. Thus, Theorem 2 implies Corollary 3 immediately.

Remark 12. We can also use the method in [Wang 2011, Section 4] to construct a rotationally nonsymmetric convex compact ancient solution for generalized curve shortening flow with power $\alpha \in\left(\frac{1}{2}, 1\right)$. Indeed, for mean curvature flow, a rotationally nonsymmetric convex compact ancient solution is constructed in Lemmas 4.1-4.4 of that reference. By examining the proofs of these lemmas, we can see that they work well for the generalized curve shortening flow considered here.

\section{Two-dimensional entire convex translating solution}

In this section, by using the previous results and a delicate iteration argument, we prove that under some extra condition on the asymptotic behavior of the solution at infinity the translating solution must be rotationally symmetric.

First of all, we would like to point out that instead of using Gage and Hamilton's exponential convergence of the curve shortening flow [1986], we need to use the corresponding exponential convergence for the generalized curve shortening flow and we will state it as a lemma, corresponding to [Wang 2011, Lemma 3.2].

Lemma 13. Let $\left\{\ell_{t}\right\}$ be a convex solution to the generalized curve shortening flow with initial curve $\left\{\ell_{0}\right\}$ uniformly convex. Suppose $\left\{\ell_{0}\right\}$ is in the $\delta_{0}$-neighborhood of a unit circle, $\left\{\ell_{t}\right\}$ shrinks to the origin at $t=\frac{1}{1+\alpha}$. Let

$$
\tilde{\ell}_{t}=(1-(1+\alpha) t)^{-\frac{1}{1+\alpha}} \ell_{t}
$$

be the normalization of $\ell_{t}$. Then $\tilde{\ell}_{t}$ is in the $\delta_{t}$-neighborhood of the unit circle centered at the origin,

$$
\tilde{\ell}_{t} \subset N_{\delta_{t}} S^{1}
$$

with

$$
\delta_{t} \leq C \delta_{0}\left(\frac{1}{1+\alpha}-t\right)^{\iota}
$$

for some small positive constant $\iota$.

Remark 14. Exponential convergence of the standard curve shortening flow (when $\alpha=1$ ) was proved by Gage and Hamilton [1986]. For the general case (when $\alpha>\frac{1}{3}$ ), as discussed in the following proof, Gage and Hamilton's method combined with Andrews' estimates [1998, Propositions II1.1 and II1.2] can still be used to prove the corresponding exponential convergence result.

Proof. The proof of Lemma 13 is similar to the proof of [Wang 2011, Lemma 3.2]. Since the initial curve $\ell_{0}$ is uniformly convex and close to a unit circle, by [Andrews 1998, Propositions II1.1 and II1.2], the curvature of $\tilde{\ell}_{t}$ is bounded from below and from above by some constant depending only on $\delta_{0}$, when $t \in\left(\frac{1}{4 \alpha+4}, \frac{1}{2 \alpha+2}\right)$. 
Hence the evolution equation for $\tilde{\ell}_{t}$ is uniformly parabolic. Therefore, we can apply Schauder's estimates safely for $\alpha>\frac{1}{2}$, as in [Wang 2011], which says that

$$
\left\|\tilde{\ell}_{t}-S^{1}\right\|_{C^{k}} \leq C \delta_{0} \quad \text { for } t \in\left(\frac{1}{4 \alpha+4}, \frac{1}{2 \alpha+2}\right) .
$$

Although the constant $C$ will depend on the lower and upper bounds of the curvature of the initial curve, it is not a problem for our purpose, since when we blow down the solution for $\sigma=0$, the norm of the gradient $D u_{h}$ on the curve $\left\{u_{h}(x)=1\right\}$ approaches 1 .

By the equation $\kappa u_{\gamma}^{\frac{1}{\alpha}}=1$, we see that the curvature $\kappa$ is also very close to 1 on that curve. For the exponential decay rate of the derivative of curvature, one can imitate the proof in [Gage and Hamilton 1986, §§5.7.10-5.7.15], and our corresponding estimate will be $\left|\kappa^{\prime}(\tau)\right| \leq C \delta_{0} e^{-\iota \tau}$ for some small positive number $\iota$, where $\tau=-\frac{1}{1+\alpha} \log \left(\frac{1}{1+\alpha}-t\right)$. Indeed, in the case $\alpha>1$, this is done by Chen and Huang [Huang 2011, Corollary 3.2], and it is easy to check that their computation also works for the case $\frac{1}{3}<\alpha<1$ by taking $\ell$ small enough. This estimate immediately implies our lemma.

In the following we will consider the case when $\sigma=1$ and $\alpha>1$. By translating and adding some constant we can assume $u(0)=\inf u$. Let

$$
u_{h}(x)=\frac{1}{h} u\left(h^{\frac{1}{1+\alpha}} x\right) .
$$

Then $u_{h}$ satisfies the equation $L_{\sigma} u_{h}=1$ with $\sigma=h^{-\frac{2 \alpha}{1+\alpha}}$. By Theorem $1, u_{h}$ converges to $\frac{1}{1+\alpha}|x|^{1+\alpha}$, and the level set $\Gamma_{\frac{1}{1+\alpha}}, u_{h}$ converges to the unit circle as $h \rightarrow \infty$.

Lemma 15 [Wang 2011, Lemma 3.3]. The function u satisfies

$$
u(x)=\frac{1}{1+\alpha}|x|^{1+\alpha}+O\left(|x|^{1+\alpha-2 \alpha \beta}\right),
$$

where $\beta$ is a constant, chosen such that $\frac{1}{2 \alpha}<\beta<\min \left\{1, \frac{1+\alpha}{2 \alpha}\right\}$.

Proof. For any given small $\delta_{0}>0$, take $h$ sufficiently large such that

$$
\Gamma_{\frac{1}{1+\alpha}, u_{h}} \subset N_{\delta_{0}}\left(S^{1}\right)
$$

for the unit circle $S^{1}$ with center $p_{0}$. Note that when $h$ is large, $\delta_{0}$ is very close to 0 . Then we will prove the following claim:

Claim 3: For small fixed $\tau$,

$$
\Gamma_{\tau, u_{h}} \subset((1+\alpha) \tau)^{\frac{1}{1+\alpha}} N_{\delta_{\tau}}\left(\left(1+\frac{a_{0}}{\tau}\right)^{\frac{1}{1+\alpha}} S^{1}\right)
$$

with

$$
\delta_{\tau} \leq C_{1}(\tau) \sigma^{\beta}+C_{2} \delta_{0} \tau^{\eta}
$$


where $\eta$ is a small positive constant, the constants $C_{1}$ and $C_{2}$ are independent of $\delta_{0}$ and $h$, and $C_{2}$ is also independent of $\tau$. Let $u_{0}$ be the solution of

satisfying

$$
L_{0}(u)=1 \quad \text { in } \Omega_{\frac{1}{1+\alpha}}, u_{h},
$$

$$
u_{0}=u_{h}=\frac{1}{1+\alpha} \quad \text { on } \partial \Omega_{\frac{1}{1+\alpha}}, u_{h},
$$

where $a_{0}=\left|\inf u_{0}\right|$ and the center of

$$
\left(1+\frac{a_{0}}{\tau}\right)^{\frac{1}{1+\alpha}} S^{1}
$$

is the minimum point of $u_{0}$ multiplied by the factor $((1+\alpha) \tau)^{-\frac{1}{1+\alpha}}$.

Proof of Claim 3. We only need to prove that

$$
\operatorname{dist}\left((1+\alpha)^{\frac{1}{1+\alpha}}\left(\tau+a_{0}\right)^{\frac{1}{1+\alpha}} S^{1}, \Gamma_{\tau, u}\right) \leq C_{1}(\tau) \sigma^{\beta}+C_{2} \delta_{0} \tau^{\frac{1}{1+\alpha}+\eta},
$$

where $\eta$ is some small positive constant and $C_{2}$ is independent of $\tau$. By Theorem 1 we know that $u_{h}$ converges to $\frac{1}{1+\alpha}|x|^{1+\alpha}$ uniformly on any compact subset of $\mathbb{R}^{2}$. Then by the convexity of $u_{h}$, we have that $\left|D u_{h}\right|$ is bounded above and below by some constants depending on $\tau_{0}$ for large $h$ when

$$
x \in\left\{x \in \Omega_{\frac{1}{1+\alpha}, u_{h}}: \tau_{0} \leq u_{h}<\frac{1}{1+\alpha}\right\} .
$$

Hence, by the growth condition for $D^{2} u$ in Theorem 2, we have $\sigma\left(u_{h}\right)_{\gamma \gamma} \leq C \sigma^{\beta}$, where $C$ is a constant depending on $\tau_{0}$. Therefore, we have

$$
\kappa\left(u_{h}\right)_{\gamma}^{1 / \alpha} \approx 1-C \sigma^{\beta} \text { on }\left\{x \in \Omega_{\frac{1}{1+\alpha}, u_{h}}: \tau \leq u_{h}<\frac{1}{1+\alpha}\right\},
$$

where $C$ depends on $\tau_{0}$. Let

$$
\tilde{u}_{0}=\left(1-C \sigma^{\beta}\right)^{\alpha}\left(u_{0}-\frac{1}{1+\alpha}\right)+\frac{1}{1+\alpha} ;
$$

then

with

$$
L_{0}\left(\tilde{u}_{0}\right)=1-C \sigma^{\beta} \quad \text { in } \Omega_{\frac{1}{1+\alpha}}, u_{h}
$$

$$
\tilde{u}_{0}=u_{h}=\frac{1}{1+\alpha} \quad \text { on } \partial \Omega_{\frac{1}{1+\alpha}}, u_{h} .
$$

Now by the comparison principle, $\Omega_{\tau, u_{0}} \supset \Omega_{\tau, u_{h}} \supset \Omega_{\tau, \tilde{u}_{0}}$, and by the asymptotic behavior of $u_{0}$,

$$
\Gamma_{\tau, u_{0}} \subset N_{\zeta}\left(\left(\tau+a_{0}\right)^{\frac{1}{1+\alpha}} S^{1}\right) \quad \text { and } \quad \Gamma_{\tau, \tilde{u}_{0}} \subset N_{\zeta}\left(\left(\tau+a_{0}-C \sigma^{\beta}\right)^{\frac{1}{1+\alpha}} S^{1}\right),
$$

where $\zeta=C \delta_{0}\left(\tau+a_{0}\right)^{\eta}$. Let

$$
\ell_{1}=\left(\tau+a_{0}\right)^{\frac{1}{1+\alpha}} S^{1} \text { and } \quad \ell_{2}=\left(\tau+a_{0}-C \sigma^{\beta}\right)^{\frac{1}{1+\alpha}} S^{1},
$$


both centered at $p_{1}$, which is the minimum point of $u_{0}$. Hence

$$
\operatorname{dist}\left(\left(\tau+a_{0}\right)^{\frac{1}{1+\alpha}} S^{1}, \Gamma_{\tau, u_{h}}\right) \leq \operatorname{dist}\left(\ell_{1}, \ell_{2}\right)+C \delta_{0}\left(\tau+a_{0}\right)^{\frac{1}{1+\alpha}+\eta},
$$

where $\operatorname{dist}\left(\ell_{1}, \ell_{2}\right)$ can be bounded by $C_{1}(\tau) \sigma^{\beta}$; hence (60) follows from the above discussion.

Now, we will use an iteration argument to prove the following claim, which will enable us to simplify (59) and (60).

Claim 4: $a_{0} \leq C \sigma^{\beta}|\log (\sigma)|$

Proof. We fix a large constant $A$ such that $\left\{u_{A / \tau}=\frac{1}{1+\alpha}\right\}$ is very close to a unit circle. Let $u_{0, \tau^{k}}$ solve $L_{0} u=1$ with boundary condition $u=\tau^{k}$ on $\left\{u_{h}=\tau^{k}\right\}$. Denote $a_{k}=\left|\inf u_{0, \tau^{k}}\right|$. From the proof of Claim 3, we see that

$$
\left\{u_{0}<\tau\right\} \supset\left\{u_{0, \tau}<\tau\right\} \supset\left\{\tilde{u}_{0}<\tau\right\},
$$

by the comparison principle, we have inf $u_{0}<\inf u_{0, \tau}<\inf \tilde{u}_{0}$. So by the construction of $\tilde{u}_{0}$ and a simple computation, we have $a_{0}-a_{1} \leq \inf \tilde{u}_{0}-\inf u_{0} \leq C \sigma^{\beta}$. When $\tau^{k} \geq \frac{A}{h}$, we can iterate this argument for $u_{0, \tau^{k}}$ and $u_{0, \tau^{k+1}}$ by rescaling them to

$$
\frac{1}{1+\alpha} \tau^{-k} u_{0, \tau^{k}}\left((1+\alpha)^{\frac{1}{1+\alpha}} \tau^{\frac{k}{1+\alpha}} x\right) \text { and } \quad \frac{1}{1+\alpha} \tau^{-k} u_{0, \tau^{k+1}}\left((1+\alpha)^{\frac{1}{1+\alpha}} \tau^{\frac{k}{1+\alpha}} x\right) \text {, }
$$

respectively. After rescaling back, we have $a_{k}-a_{k+1} \leq C \sigma^{\beta}$. Note that the choice of $A$ and the condition $\tau^{k} \geq \frac{A}{h}$ ensure the uniform gradient bound needed in the above argument. Let $k_{0}$ be an integer satisfying $\tau^{k_{0}} \geq \frac{A}{h} \geq \tau^{k_{0}+1}$. After $k_{0}$ steps we stop the iteration, and notice that

$$
\left\{u_{h}=\frac{A}{h}\right\}=h^{-\frac{1}{1+\alpha}}\{u=A\}
$$

is contained in a circle with radius $C h^{-\frac{1}{1+\alpha}}$ for some constant $C$. Hence it takes at most time $C h^{-1}=C \sigma^{\frac{1+\alpha}{2 \alpha}}$ for $\left\{u_{h}=\frac{A}{h}\right\}$ to shrink to a point. Claim 4 follows from the above discussion.

By omitting the lower order term we can rewrite (59) and (60) as

$$
\Gamma_{\tau, u_{h}} \subset((1+\alpha) \tau)^{\frac{1}{1+\alpha}} N_{\delta_{\tau}}\left(S^{1}\right)
$$

with

$$
\delta_{\tau} \leq C_{1}(\tau) \sigma^{\beta}+C_{2} \delta_{0} \tau^{\eta}
$$

If we take $\tau$ small such that $C_{2} \tau^{\eta} \leq \frac{1}{4}$, then (63) becomes

$$
\delta_{\tau} \leq C_{1}(\tau) \sigma^{\beta}+\frac{1}{4} \delta_{0} .
$$


Now we can carry out an iteration argument similar to that in [Wang 2011]. We start at the level $\frac{1}{1+\alpha} \tau^{-k_{0}}$ for some sufficient large $k_{0}$. Let

$$
\Omega_{k}=\tau^{\frac{k}{1+\alpha}} \Omega_{\frac{1}{1+\alpha}} \tau^{-k}, u \quad \text { and } \quad \Gamma_{k}=\partial \Omega_{k} .
$$

Note that $\Gamma_{k}$ converges to a unit circle as $k \rightarrow \infty$. Suppose that $\Gamma_{k}$ is in the $\delta_{k}$ neighborhood of $S^{1}$ centered at $y_{k}$, where $\delta_{k} \rightarrow 0$ as $k \rightarrow \infty$ and $y_{k}$ is the minimum point of the solution of $L_{0} u=1$ in $\Omega_{k}$ with $u=\frac{1}{1+\alpha}$ on $\Gamma_{k+1}$. By (64) we have

$$
\delta_{k-1} \leq C_{1}(\tau) \tau^{(k-1) \frac{2 \alpha \beta}{1+\alpha}}+\frac{1}{4} \delta_{k}
$$

for $k=k_{0}, k_{0}+1, \ldots$ Then we have

$$
\Gamma_{j} \subset N_{\delta_{j}}\left(S^{1}\right)
$$

with

$$
\delta_{j} \leq C \tau^{j \frac{2 \alpha \beta}{1+\alpha}}
$$

It follows that

$$
\Gamma_{\frac{1}{1+\alpha}} \tau^{-j, u} \subset N_{\tilde{\delta}_{j}}\left(\tau^{\frac{-j}{1+\alpha}} S^{1}\right)
$$

with

$$
\tilde{\delta}_{j} \leq C \tau^{\frac{2 \alpha \beta-1}{1+\alpha} j}
$$

where $\tau^{\frac{-j}{1+\alpha}} S^{1}$ is centered at $z_{j}=\tau^{\frac{-j}{1+\alpha}} y_{j}$. From Lemma 13 and (64), it is not hard to see that

$$
\left|z_{j}-z_{j-1}\right| \leq C \tau^{\frac{2 \alpha \beta-1}{1+\alpha} j} .
$$

Let $z_{0}=\lim _{j \rightarrow \infty} z_{j}$. Then

$$
\left|z_{j}-z_{0}\right| \leq C \tau^{\frac{2 \alpha \beta-1}{1+\alpha} j},
$$

which means that in (68) we can assume the circle is centered at $z_{0}$ by changing the constant $C$ a little bit. In fact when we choose different $\tau$, the corresponding $z_{0}$ will not change, so we can assume $z_{0}=0$. Hence, for $h=\frac{1}{1+\alpha} \tau^{-j}$,

$$
\Gamma_{h, u} \subset N_{\delta}\left((1+\alpha)^{\frac{1}{1+\alpha}} h^{\frac{1}{1+\alpha}} S^{1}\right),
$$

where

$$
\delta \leq C h^{\frac{1-2 \alpha \beta}{1+\alpha}}
$$

and $S^{1}$ is centered at the origin. By choosing different $\tau$, we see that the estimate holds for all large $h$. Lemma 15 follows from the above estimates. 
Remark 16. For the mean curvature flow (when $\alpha=1$ ), I learned the proof of Claim 4 from Professor Xu-Jia Wang. Indeed, in this case one can do it as follows. Let $v_{k}$ solve $L_{0} w=1$ with boundary condition $w=\tau^{k}$ on $\left\{u_{h}=\tau^{k}\right\}$, for $k=1,2, \ldots$ Let $a_{k}=\left|\inf v_{k}\right|$. Then by [Wang 2011, Lemma 3.1], we have $a_{0}-a_{1} \leq C \sigma$. By rescaling invariance, we can iterate the argument to show that $a_{k}-a_{k+1} \leq C \sigma$, provided $\tau^{k} \geq \frac{1}{h}$. Hence, we stop the iteration at $k_{0}$ when $\tau^{k_{0}} \geq \frac{1}{h}>\tau^{k_{0}+1}$. Notice that

$$
\left\{u_{h} \leq \tau^{k_{0}}\right\}=h^{-\frac{1}{2}}\left\{u=h \tau^{k_{0}}\right\} \subset h^{-\frac{1}{2}}\left\{u \leq \frac{1}{\tau}\right\} .
$$

So, it is easy to see that it takes at most time $C \sigma=\frac{C}{h}$ for $\left\{u_{h} \leq \tau^{k_{0}}\right\}$ to shrink to a point, namely, $a_{k_{0}} \leq C \sigma$. Therefore,

$$
a_{0}=a_{k_{0}}+\sum_{i=0}^{k_{0}-1} a_{i}-a_{i+1} \leq C k_{0} \sigma \leq C \sigma|\log \sigma| .
$$

In order to finish the proof of Theorem 2 we need to use the following fundamental Liouville theorem by Bernstein [Simon 1997, p. 245].

Lemma 17. Let $u$ be an entire solution to the elliptic equation

$$
\sum_{i, j=1}^{n} a_{i j}(x) u_{i j}=0 \quad \text { in } \mathbb{R}^{2} .
$$

If $u$ satisfies the asymptotic estimate

$$
|u(x)|=o(|x|) \quad \text { as } x \rightarrow \infty,
$$

then $u$ is a constant.

Proof of the second part of Theorem 2. Let $u^{*}$ be the Legendre transform of $u$. Then $u^{*}$ satisfies equation

$$
G\left(x, D^{2} u^{*}\right)=\frac{\operatorname{det} D^{2} u^{*}}{\left(\delta_{i j}-\frac{x_{i} x_{j}}{1+|x|^{2}}\right) F^{i j}\left(u^{*}\right)}=\left(1+|x|^{2}\right)^{\frac{1}{2 \alpha}-\frac{1}{2}},
$$

where $F^{i j}\left(u^{*}\right)=\frac{\partial \operatorname{det} r}{\partial r_{i j}}$ at $r=D^{2} u^{*}$. We have

$$
u^{*}(x)=C(\alpha)|x|^{1+\alpha}+O\left(|x|^{\frac{1+\alpha-2 \alpha \beta}{\alpha}}\right),
$$

where $C(\alpha)$ is a constant depending only on $\alpha$. In fact, for big $h$, by Lemma 15 ,

$$
u_{h}(x)=\frac{1}{1+\alpha}|x|^{1+\alpha}+O\left(|h|^{\frac{-2 \alpha \beta}{1+\alpha}}\right)
$$

in $B_{1}(0)$. Denote by $u_{h}^{*}$ the Legendre transform of $u_{h}$. Then,

$$
u_{h}^{*}(x)=C(\alpha)|x|^{1+\frac{1}{\alpha}}+O\left(|h|^{\frac{-2 \alpha \beta}{1+\alpha}}\right),
$$


where $C(\alpha)$ is a constant depending only on $\alpha$ and it comes from the Legendre transform of the function $\frac{1}{1+\alpha}|x|^{1+\alpha}$. Note that $u_{h}^{*}(x)=h^{-1} u^{*}\left(h^{\frac{\alpha}{1+\alpha}} x\right)$, we obtain (74).

Let $u_{0}$ be the unique radial solution of (3) with $\sigma=1$, and let $u_{0}^{*}$ be the Legendre transform of $u_{0}$. Similar to (74) we have

$$
u_{0}^{*}(x)=C(\alpha)|x|^{1+\alpha}+O\left(|x|^{\frac{1+\alpha-2 \alpha \beta}{\alpha}}\right) .
$$

Since both $u^{*}$ and $u_{0}^{*}$ satisfy (73), $v=u^{*}-u_{0}^{*}$ satisfies the elliptic equation

$$
\sum_{i, j=1}^{n} a_{i j}(x) v_{i j}=0 \quad \text { in } \mathbb{R}^{2},
$$

where

$$
a_{i j}=\int_{0}^{1} G^{i j}\left(x, D^{2} u_{0}^{*}+t\left(D^{2} u^{*}-D^{2} u_{0}^{*}\right)\right) d t
$$

Here,

$$
G^{i j}=\frac{\partial G(x, r)}{\partial r_{i j}}
$$

for any symmetric matrix $r$. Note that by the choice of $\beta, \frac{1+\alpha-2 \alpha \beta}{\alpha}<1$; hence, by (74) and (75),

$$
v=O\left(|x|^{\frac{1+\alpha-2 \alpha \beta}{\alpha}}\right)=o(|x|) \quad \text { as }|x| \rightarrow \infty .
$$

By Lemma 17 we conclude that $v$ is a constant.

\section{References}

[Andrews 1998] B. Andrews, "Evolving convex curves", Calc. Var. PDE 7:4 (1998), 315-371. MR 99k:58038 Zbl 0931.53030

[Andrews 2002] B. Andrews, "Non-convergence and instability in the asymptotic behaviour of curves evolving by curvature", Comm. Anal. Geom. 10:2 (2002), 409-449. MR 2003e:53086 Zbl 1029.53079

[Andrews 2003] B. Andrews, "Classification of limiting shapes for isotropic curve flows", J. Amer. Math. Soc. 16:2 (2003), 443-459. MR 2004a:53083 Zbl 1023.53051

[Chou and Zhu 2001] K.-S. Chou and X.-P. Zhu, The curve shortening problem, Chapman \& Hall/CRC, Boca Raton, FL, 2001. MR 2003e:53088 Zbl 1061.53045

[Daskalopoulos et al. 2010] P. Daskalopoulos, R. Hamilton, and N. Sesum, "Classification of compact ancient solutions to the curve shortening flow", J. Differential Geom. 84:3 (2010), 455-464. MR 2012d:53213 Zbl 1205.53070

[Gage and Hamilton 1986] M. Gage and R. S. Hamilton, "The heat equation shrinking convex plane curves”, J. Differential Geom. 23:1 (1986), 69-96. MR 87m:53003 Zbl 0621.53001

[Huang 2011] R. L. Huang, "Blow-up rates for the general curve shortening flow", J. Math. Anal. Appl. 383:2 (2011), 482-489. MR 2012h:53152 Zbl 1220.53082 
[Kawohl 1985] B. Kawohl, Rearrangements and convexity of level sets in PDE, Lecture Notes in Mathematics 1150, Springer, Berlin, 1985. MR 87a:35001 Zbl 0593.35002

[Schulze 2005] F. Schulze, "Evolution of convex hypersurfaces by powers of the mean curvature", Math. Z. 251:4 (2005), 721-733. MR 2006h:53065 Zbl 1087.53062

[Sheng and Wu 2009] W. Sheng and C. Wu, "On asymptotic behavior for singularities of the powers of mean curvature flow", Chin. Ann. Math. (B) 30:1 (2009), 51-66. MR 2010h:53100 Zbl 1180.53066

[Simon 1997] L. Simon, “The minimal surface equation”, pp. 239-266 in Geometry V, edited by R. Osserman, Encyclopaedia of Mathematical Sciences 90, Springer, Berlin, 1997. MR 99b:53014 Zbl 0905.53003

[Wang 2011] X.-J. Wang, "Convex solutions to the mean curvature flow", Ann. of Math. (2) 173:3 (2011), 1185-1239. MR 2800714 Zbl 1231.53058

[White 2000] B. White, "The size of the singular set in mean curvature flow of mean-convex sets", $J$. Amer. Math. Soc. 13:3 (2000), 665-695. MR 2001j:53098 Zbl 0961.53039

Received February 19, 2013. Revised October 19, 2014.

\section{SHIBING CHEN}

DEPARTMENT OF MATHEMATICS

ZHEJIANG UNIVERSITY OF TECHNOLOGY

HANGZHOU, ON 310023

CHINA

Current address:

Mathematical Sciences Institute

The Australian National University

JOHN DEDMAN BUILDING 27

CANBERRA ACT 2601

Australia

chenshibing1982@hotmail.com 


\title{
PACIFIC JOURNAL OF MATHEMATICS
}

\author{
msp.org/pjm
}

Founded in 1951 by E. F. Beckenbach (1906-1982) and F. Wolf (1904-1989)

\section{EDITORS}

Don Blasius (Managing Editor)

Department of Mathematics

University of California

Los Angeles, CA 90095-1555

blasius@math.ucla.edu

\author{
Paul Balmer \\ Department of Mathematics \\ University of California \\ Los Angeles, CA 90095-1555 \\ balmer@math.ucla.edu \\ Robert Finn \\ Department of Mathematics \\ Stanford University \\ Stanford, CA 94305-2125 \\ finn@math.stanford.edu \\ Sorin Popa \\ Department of Mathematics \\ University of California \\ Los Angeles, CA 90095-1555 \\ popa@math.ucla.edu
}

\author{
Vyjayanthi Chari \\ Department of Mathematics \\ University of California \\ Riverside, CA 92521-0135 \\ chari@math.ucr.edu \\ Kefeng Liu \\ Department of Mathematics \\ University of California \\ Los Angeles, CA 90095-1555 \\ liu@math.ucla.edu \\ Jie Qing \\ Department of Mathematics \\ University of California \\ Santa Cruz, CA 95064 \\ qing@ cats.ucsc.edu
}

\section{PRODUCTION}

Silvio Levy, Scientific Editor, production@msp.org

\section{SUPPORTING INSTITUTIONS}

ACADEMIA SINICA, TAIPEI

CALIFORNIA INST. OF TECHNOLOGY

INST. DE MATEMÁTICA PURA E APLICADA

KEIO UNIVERSITY

MATH. SCIENCES RESEARCH INSTITUTE

NEW MEXICO STATE UNIV.

OREGON STATE UNIV.

\author{
STANFORD UNIVERSITY \\ UNIV. OF BRITISH COLUMBIA \\ UNIV. OF CALIFORNIA, BERKELEY \\ UNIV. OF CALIFORNIA, DAVIS \\ UNIV. OF CALIFORNIA, LOS ANGELES \\ UNIV. OF CALIFORNIA, RIVERSIDE \\ UNIV. OF CALIFORNIA, SAN DIEGO \\ UNIV. OF CALIF., SANTA BARBARA
}

\author{
Daryl Cooper \\ Department of Mathematics \\ University of California \\ Santa Barbara, CA 93106-3080 \\ cooper@math.ucsb.edu \\ Jiang-Hua Lu \\ Department of Mathematics \\ The University of Hong Kong \\ Pokfulam Rd., Hong Kong \\ jhlu@maths.hku.hk \\ Paul Yang \\ Department of Mathematics \\ Princeton University \\ Princeton NJ 08544-1000 \\ yang@math.princeton.edu
}

These supporting institutions contribute to the cost of publication of this Journal, but they are not owners or publishers and have no responsibility for its contents or policies.

See inside back cover or msp.org/pjm for submission instructions.

The subscription price for 2015 is US \$420/year for the electronic version, and \$570/year for print and electronic.

Subscriptions, requests for back issues and changes of subscribers address should be sent to Pacific Journal of Mathematics, P.O. Box 4163, Berkeley, CA 94704-0163, U.S.A. The Pacific Journal of Mathematics is indexed by Mathematical Reviews, Zentralblatt MATH, PASCAL CNRS Index, Referativnyi Zhurnal, Current Mathematical Publications and Web of Knowledge (Science Citation Index).

The Pacific Journal of Mathematics (ISSN 0030-8730) at the University of California, c/o Department of Mathematics, 798 Evans Hall \#3840, Berkeley, CA 94720-3840, is published twelve times a year. Periodical rate postage paid at Berkeley, CA 94704, and additional mailing offices. POSTMASTER: send address changes to Pacific Journal of Mathematics, P.O. Box 4163, Berkeley, CA 94704-0163.

PJM peer review and production are managed by EditFLOW ${ }^{\circledR}$ from Mathematical Sciences Publishers.

\section{PUBLISHED BY}

\section{mathematical sciences publishers \\ nonprofit scientific publishing}

http://msp.org/

(C) 2015 Mathematical Sciences Publishers 


\section{PACIFIC JOURNAL OF MATHEMATICS}

Volume $276 \quad$ No. $1 \quad$ July 2015

On the degree of certain local $L$-functions

U. K. ANANDAVARdhanan and AmiYa Kumar MondaL

Torus actions and tensor products of intersection cohomology

ASILATA BAPAT

Cyclicity in Dirichlet-type spaces and extremal polynomials II: functions on the bidisk

Catherine Bénéteau, Alberto A. Condori, Constanze Liaw,

DANIEl SECo and Alan A. SOlA

Compactness results for sequences of approximate biharmonic maps

CHRISTINE BREINER and TOBIAS LAMM

Criteria for vanishing of Tor over complete intersections

Olgur Celikbas, Srikanth B. Iyengar, Greg PiePMeyer and

ROGER WIEGAND

Convex solutions to the power-of-mean curvature flow

\section{SHIBING CHEN}

Constructions of periodic minimal surfaces and minimal annuli in $\mathrm{Sol}_{3}$

CHRISTOPHE DESMONTS

Quasi-exceptional domains

ALEXANDRE EREMENKO and ERIK LUNDBERG

Endoscopic transfer for unitary groups and holomorphy of Asai $L$-functions

NEVEN GRBAC and FREYDOON SHAHIDI

Quasiconformal harmonic mappings between Dini-smooth Jordan domains

DAVID KALAJ

Semisimple super Tannakian categories with a small tensor generator

THOMAS KRÄMER and RAINER WEISSAUER

On maximal Lindenstrauss spaces

PETR PETRÁČEK and JiŘí SPURNÝ 